\title{
$L$ FUNCTIONS FOR THE GROUP $G_{2}$
}

\author{
I. PIATETSKI-SHAPIRO, S. RALLIS, AND G. SCHIFFMANN
}

\section{INTRODUCTION}

The method of $L$ functions is one of the major methods for analyzing automorphic forms. For example, the Hecke Converse Theorem gives an equivalence via the Mellin transform between holomorphic modular forms on the upper half plane and certain $L$ functions associated to Dirichlet series, which have analytic continuation and functional equation. The classical theory of automorphic forms on the group $G L_{2}$ can be reinterpreted in terms of the spectral analysis of functions on the space $G L_{2}(k) \backslash G L_{2}(\mathbf{A})$ where $\mathbf{A}=\mathbf{A}_{k}$ is the adele ring of the number field $k$, ([W-1]). Using adelic language, Weil and Jacquet-Langlands have developed the Hecke Converse Theorem for $G L_{2}$ from a representation theoretic point of view ([W-2, J-L]). This leads to the problem of analyzing the class of automorphic representations of a reductive group, $G$. That is, we consider a $G(\mathbf{A})$ irreducible representation $\Pi$ embedded in a suitable subspace of $G(k) \backslash G(\mathbf{A})$. By the general theory developed by Langlands ([L]) one can associate to $\Pi$ a whole class of $L$ functions parametrized by the finite-dimensional modules of an associated $L$ group. The first two major questions that arise are whether these automorphic $L$ functions have analytic continuation and functional equation. These questions are particularly relevant in that such automorphic $L$ functions are closely tied to applications in number theory. For instance, it is expected from Langlands' philosophy that the $L$ functions describing the arithmetic structure of certain algebraic varieties can be described in terms of automorphic $L$ functions.

The problem of determining the analytic continuation and functional equation of general $L$ functions associated to automorphic representations of reductive Lie groups and representations of the

Received by the editors March 29, 1989 and, in revised form, January 2, 1990. 1980 Mathematics Subject Classification(1985 Revision). Primary 11F66, 11 F70.

The first author was supported by NSF grant DMS-8400139.

The second author was supported by NSF grant DMS- 8401947 . 
$L$ groups has been pursued by two different techniques. The first is the interpretation of the $L$ function as arising in the constant term of Eisenstein series (the Langlands-Shahidi method). The second is the generalized Rankin-Selberg integral representation of the $L$ function. We refer the reader to [GS] for an excellent description of both methods and a comparison of the various techniques used.

The second method was originally developed to study the analytic properties of the $L$ function associated to the tensor product of automorphic representations of $G L_{2}(\mathbf{A})$. The nonvanishing of such Rankin-Selberg $L$ functions on the line $\operatorname{Re}(s)=1$ implies certain estimates on the Hecke eigenvalues of the local components of an automorphic cuspidal representation of $G L_{2}(\mathbf{A})$. In concrete terms this determines the location of an unramified local component of a global automorphic representation in terms of how close to the end of the complementary series such a representation must be.

The next step in the development of the Rankin-Selberg $L$ functions is the work (J-PS-Sh). Namely here the tensor product of two automorphic representations of $G L_{n}$ and $G L_{m}$ is considered. The main use of such work is twofold. First, one can prove a Strong Multiplicity One Theorem for cusp forms on $G L_{n}(\mathbf{A})$. This means simply that two global cuspidal $G L_{n}(\mathbf{A})$ representations $\Pi=\bigotimes_{v} \Pi_{v}$ and $\sigma=\bigotimes_{v} \sigma_{v}$ (with the same central character) are equal if and only if $\Pi_{v} \cong \sigma_{v}$ for almost all $v$. Second, the Rankin-Selberg $L$ function is used to determine the predicted classification of automorphic representations of $G L_{n}(\mathbf{A})$. That is, each automorphic representation is given as a subquotient of an induced module with cuspidal data, where such data is determined uniquely up to associate class [J-S-1,2].

Subsequently in [G-PS-R] a theory for Rankin products is developed for groups of the form $G L_{n} \times G^{\prime}, G^{\prime}$ a classical group. The specific example of the triple tensor product of $G L_{2}$ automorphic representations is given in [PS-R].

The common feature of the above examples is a setup of the following form. Namely we are given three reductive groups $G_{1}$, $G$, and $H$ so that $G \supset G_{1}$ and $H \supset G_{1}$. Then we consider the corresponding adelic groups $G(\mathbf{A}), G_{1}(\mathbf{A})$, and $H(\mathbf{A})$. We let $f_{\Pi_{1}} \in \Pi_{1}$, a cuspidal irreducible representation of $G(\mathbf{A})$. If $P_{H}$ is a maximal parabolic subgroup of $H$, then $P_{H}=M_{H} \cdot U_{H}$ where $M_{H}$ is a Levi subgroup and $U_{H}$ is the unipotent radical 
of $P_{H}$. We let $\delta_{P_{H}}$ be the corresponding modular function associated to the adjoint action of $P_{H}$ on its Lie algebra. Then $\delta_{P_{H}}$ is a character on $P_{H}$. We let $\Pi_{2}$ be a cuspidal representation of $M_{H}(\mathbf{A})$. Then with this data we form the induced module $\operatorname{ind}_{P_{H}(\mathbf{A})}^{H(\mathbf{A})}\left(\Pi_{2} \otimes\left|\delta_{P_{H}}\right|^{s}\right)$ with $s \in \mathbf{C}$. From this we can form a family of Einsenstein series $\mathscr{E}_{s}\left(\Pi_{2}, x\right)$ on the group $H(\mathbf{A})$. Then we form the integral

$$
\int_{G_{1}(k) \backslash G_{1}(\mathbf{A})} f_{\Pi_{1}}\left(g_{1}\right) \mathscr{E}_{s}\left(\Pi_{2}, g_{1}\right) d g_{1} .
$$

The problem is to determine when such an integral represents a good $L$ function, i.e., one that is an Euler product and is related to $\Pi_{1}$ and $\Pi_{2}$ in a simple way. In any event, the above integral as a function in $s$ has a meromorphic continuation and a functional equation with a precise location of poles. This follows from knowing the same information about the Eisenstein series $\mathscr{E}_{s}\left(\Pi_{2},\right)$; this in turn follows from the Langlands-Shahidi method mentioned above. In any case the analytic $L$ function that arises from the integral should be more complicated than the $L$ function that arises in normalizing the Eisenstein series $\mathscr{E}_{s}\left(\Pi_{2}, \quad\right)$.

In any event the Langlands-Shahidi method determines a prescribed list of $L$ functions; this list does not include all the possible cases of $L$ functions in [L]. The Rankin-Selberg method has so far yielded only some of the $L$ functions that come about by the first method. Thus, one of the major question is whether the Rankin-Selberg method will give new examples of $L$ functions not possible by Langlands-Shahidi method. We show that, in fact, this is the case. We construct here, via the Rankin-Selberg method, the $L$ function associated to the tensor product of the standard 7-dimensional representation of $G_{2}$ with the standard two-dimensional representation of $G L_{2}$. In fact, as a bonus of our method, we also represent the $L$ function of the standard 7-dimensional representation of $G_{2}$ !

\section{DATA OF PROBlem}

Let $k$ be a field of characteristic 0 . Let $\Lambda^{*}\left(k^{m}\right)$ be the exterior algebra over the $m$-dimensional column space $k^{m}$. We consider, specifically, the case $m=7$ and the subspace $\Lambda^{3}\left(k^{7}\right)$. The group $G L_{7}(k)$ acts on $k^{7}$ (thru left multiplication of matrices). We consider the action of $G L_{7}$ on $\Lambda^{*}$ and, in particular, on $\Lambda^{3}\left(k^{7}\right)$. 
We say a form $\psi \in \Lambda^{3}$ is nondegenerate if $\psi$ has the following property: For all $y \in k^{7}-0, \psi_{y}=\psi(y, *, *)$, (viewed as an element in $\left.\Lambda^{2}\left(k^{7} / k \cdot y\right)\right)$, is a nondegenerate 2 form. The set of all nondegenerate forms in $\Lambda^{3}$ is a stable under $G L_{7}$, and the stabilizer of any such form is a group isomorphic to $G \times Z_{3}^{*}$ with $G$, a group isomorphic to a form of $G_{2}$, where the group $Z_{3}^{*}=\mathbf{Z} / 3 \mathbf{Z}$ or 1 depending on whether $k$ contains cube roots of identity. Such a $\psi$ as above determines a symmetric form on $k^{7}$ via the following recipe: $\psi \wedge \psi_{y} \wedge \psi_{z}$ is a multiple of a fixed element $\omega_{0}$ in $\Lambda^{7}\left(k^{7}\right)$, i.e. $\psi \wedge \psi_{y} \wedge \psi_{z}=\varphi_{\psi}(y, z) \omega_{0}$. Then $\varphi_{\psi}$ determines a nondegenerate symmetric form on $k^{7}$ and $G \subseteq \operatorname{Stab}_{G L_{7}}(\psi)$ leaves $\varphi_{\psi}$ invariant. Thus $G \subseteq S O\left(\varphi_{\psi}\right)=$ the special orthogonal group of $\varphi_{\psi}$. (See [H] for these facts.)

If $k$ is either a number field or a local field then there are at most two possibilities for $\varphi_{\psi}$. Either $\varphi_{\psi}$ is anisotropic or $\varphi_{\psi}$ represents a quadratic form of Witt index 3 .

We let $X_{l}\left(\varphi_{\psi}\right)=$ the set of all isotropic subspaces of dimension equal to $l$ relative to the form $\varphi_{\psi}$. The group $G$ acts almost transitively on $X_{l}\left(\varphi_{\psi}\right)$ in the following sense.

Lemma 1.1. If $l=1$, then $G$ acts transitively in $X_{1}\left(\varphi_{\psi}\right)$. If $l=2$ or $l=3, G$ has a finite number of orbits in $X_{l}\left(\varphi_{\psi}\right)$.

Remark. We note that if $k$ is algebraically closed, the group $G$ has a finite number of orbits acting on the space $B \backslash S O\left(\varphi_{\psi}\right)$, where $B$ is a Borel subgroup of $S O\left(\varphi_{\psi}\right)$. We also note the case $l=1$ is well-known, and a proof is given in [RS]. In the case $l=2$ there are two orbits.

In the case $l=2$, the small orbit has as representative stabilizer in $G$, a parabolic subgroup $Q$ of $G$. The large orbit has as representative stabilizer, a subgroup $H$, which is given as a semidirect product $G L_{2}(k) \ltimes Z$ where $Z$ is a 3-dimensional unipotent subgroup.

We know that $X_{2}(\varphi) \cong \widetilde{P} \backslash S O\left(\varphi_{\psi}\right)$ where $\widetilde{P}$ is a parabolic subgroup of $S O\left(\varphi_{\psi}\right)$ having the form $G L_{2}(k) \times S O(3) \ltimes U$ with $U$ the 7-dimensional unipotent radical. Then we have that $H=G \cap \widetilde{P}$. The structure of $H$ above is compatible with $\widetilde{P}$ in the following sense. First $Z \subseteq S O(3) \ltimes U$ and the $G L_{2}(k)$ subgroup of $H$ is isomorphic to the embedding of $G L_{2}(k)$ into $G L_{2}(k) \times S O(3)$ 
given by the map

$$
g \rightsquigarrow(g, i(\operatorname{det} g)),
$$

and $i$ is a canonical mapping of $k^{x}$ into a split torus of $S O(3)$.

\section{Global Rankin-Selberg integrals}

We let $k$ be a number field and $k_{v}=$ the local component associated to each prime $v$ in $k$. Given an algebraic object $X$ (i.e. group or affine variety) defined over $k$, we let $X(\mathbf{A})$ be the corresponding adelized space.

We consider the embedding of $G$ into $S O\left(\varphi_{\psi}\right)$ given in $\S 1$. We recall that $\widetilde{P}$ is parabolic of $S O\left(\varphi_{\psi}\right)$ with Levi factor $G L_{2} \times$ $S O(3)$. We form the corresponding adelized groups $G(\mathbf{A})$, $S O\left(\varphi_{\psi}\right)(\mathbf{A}), \widetilde{P}(\mathbf{A})$, etc.

We consider the family of characters given on $\widetilde{P}(\mathbf{A})$ via the map $g=(x, y, u) \stackrel{\|}{\Downarrow}|\operatorname{det} x|_{\mathbf{A}}^{s+2}$ with $x \in G L_{2}(\mathbf{A}), y \in S O(3)(\mathbf{A}), u \in$ $\widetilde{U}(\mathbf{A})$. Moreover, we let $\tau$ be a cuspidal irreducible representation of $G L_{2}(\mathbf{A})$ occuring in $L^{2}\left(G L_{2}(k) \widetilde{Z}(\mathbf{A}) \backslash G L_{2}(\mathbf{A})\right)$ with $\widetilde{Z}=$ the center of $G L_{2}(k)$. This means that $\tau$ has trivial central character. Then we form the family of induced automorphic representations

$$
I(\tau, s)=\operatorname{Ind}_{\widetilde{P}(\mathbf{A})}^{\operatorname{SO}(Q)(\mathbf{A})}\left(\tau \otimes \|^{s+2} \otimes 1_{S O(3)} \otimes 1_{\widetilde{U}}\right) .
$$

With such a family we can construct Eisenstein series. Let $f(\tau, s, x)$ be an analytic family in $I(\tau, s)$. Then for $s$ so that $\operatorname{Re}(s)$ is sufficiently large we form the Eisenstein series

$$
\mathscr{E}(f(\tau, s, x))=\sum_{\widetilde{P}(k) \backslash S O(Q)(k) \ni \gamma} f(\tau, s, \gamma x) .
$$

Then $\mathscr{E}()$ has a meromorphic continuation in $s$ and admits a functional equation in $s$. For this we consider the $S O(Q)(\mathbf{A})$ intertwining operator $M_{w}: I(\tau, s) \rightarrow I(\tau,-s)$ defined by

$$
M_{w}(f(\tau, s, x))=\int_{\widetilde{U}(\mathbf{A})} f(\tau, s, w u x) d u,
$$

(with $w$, the appropriate Weyl group element which sends all the positive roots to negative roots). Then the functional equation of $\mathscr{E}(f(\tau, s)$,$) is$

$$
\mathscr{E}(f(\tau, s, x))=\mathscr{E}\left(M_{w}(f(\tau, s, x))\right) .
$$


Moreover, if $\Omega$ is any finite set of primes, we denote by the restricted $L^{\Omega}$ function as the Euler product

$$
\prod_{v \notin \Omega} L_{v}(\cdots)
$$

of the appropriate local $L_{v}(\cdots)$ factors. Since the representation $I(\tau, s)=\bigotimes_{v} I_{v}\left(\tau_{v}, s\right)$ with $I_{v}\left(\tau_{v}, s\right)$, the corresponding local representation, we say $f(\tau, s$,$) is factorizable if f(\tau, s)=$, $\prod_{v} f_{v}\left(\tau_{v}, s,\right)$. Let $\Omega^{\prime}$ be the set of primes where $f_{v}\left(\tau_{v}, s,\right)$ is not the unique spherical vector in $I_{v}\left(\tau_{v}, s\right)$. Then $f(\tau, s)=$, $f^{\Omega^{\prime}}(\tau, s,) f_{\Omega^{\prime}}(\tau, s$,$) when f^{\Omega^{\prime}}=\prod_{v \notin \Omega} f_{v}\left(\tau_{v}, s, \quad\right)$ and $f_{\Omega^{\prime}}=$ $\prod_{v \in \Omega} f_{v}\left(\tau_{v}, s,\right)$. In particular we know that

$$
\begin{aligned}
M_{w}(f(\tau, s,))= & {\left[\frac{L^{\Omega^{\prime}}(\tau, s-1 / 2)}{L^{\Omega^{\prime}}(\tau, s+3 / 2)}\right]\left[\frac{L^{\Omega^{\prime}}\left(\tau, \operatorname{Sym}^{2}, 2 s\right)}{L^{\Omega^{\prime}}\left(\tau, \operatorname{Sym}^{2}, 2 s+1\right)}\right] } \\
& \times f^{\Omega^{\prime}}(\tau,-s,) \prod_{v \in \Omega}\left(M_{w}^{v}\right)\left(f_{v}\left(\tau_{v}, s,\right)\right)
\end{aligned}
$$

where $M_{w}^{v}$ is the corresponding local intertwining operator associated to $M_{w}$. Here $L^{\Omega}(\tau, s)$ and $L^{\Omega}\left(\tau, \operatorname{Sym}^{2}, s\right)$ are the restricted $L$ functions determined by the standard 2-and 3-dimensional representations of $G L_{2}(\mathbf{C})$ associated to the cuspidal representation $\tau$ of $G L_{2}(\mathbf{A})$. Then we expect from the theory of Eisenstein series that

$$
s \rightsquigarrow L^{\Omega^{\prime}}(\tau, s+3 / 2) L^{\Omega^{\prime}}\left(\tau, \operatorname{Sym}^{2}, 2 s+1\right) \mathscr{E}(f(\tau, s, x))
$$

admits a finite number of poles.

Let $\Pi$ be a cuspidal irreducible representation of $G(\mathbf{A})$. Let $F \in \Pi$ be a smooth function. We form the Rankin-Selberg integral:

$$
\begin{aligned}
\mathrm{II}(F, f(\tau, s,)) \equiv & L^{S}(\tau, s+3 / 2) L^{S}\left(\tau, \operatorname{Sym}^{2}, 2 s+1\right) \\
& \times \int_{G(k) \backslash G(\mathbf{A})} F(x) \mathscr{E}(f(\tau, s, x)) d x .
\end{aligned}
$$

In this case the set $S$ depends on $F$ and $f(\tau, s$,$) to be specified$ below.

We let $U_{0}$ be the unipotent radical of a Borel subgroup of $G$. We consider an additive character $\psi_{U_{0}}$ on the $U_{0}(k) \backslash U_{0}(\mathbf{A})$ which has the property that it is nontrivial on each root subgroup of $U_{0}(\mathbf{A})$ which corresponds to a simple root. Then we say $\Pi$ is a 
generic representation of $G L(\mathbf{A})$ if the integral

$$
\int_{U_{0}(k) \backslash U_{0}(\mathbf{A})} F(u) \psi_{U_{0}}(u) d u
$$

is nonzero for some $F \in \Pi$.

Given $F \in \Pi$ we say $F$ is factorizable in the following sense. There exists a $G(\mathbf{A})$ intertwining map $r_{\Pi}: \bigotimes_{v} \Pi_{v} \rightarrow \Pi$ and let $r_{\Pi}\left(\otimes_{v} F_{v}\right)=F$ where $F_{v}$ for almost all $v$ is the unique $G_{v}$ spherical vector in $\Pi_{v}$.

We let $L_{v} \quad\left(\Pi_{v} \otimes \tau_{v}, s\right)$ be the local Euler factor defined in the following manner. The representation $\Pi_{v}\left(\tau_{v}\right.$ resp.) corresponds to a semisimple element $A_{v}$ in ${ }^{L} G^{0}=G_{2}(\mathbf{C}) \quad\left(B_{v}\right.$ in ${ }^{L} G L_{2}^{0}=G L_{2}(\mathbf{C})$ resp.) determined by the Satake classification of spherical representations of $G$. $\left(G L_{2}\right.$ resp.). Then $L_{v}\left(\Pi_{v} \otimes \tau_{v}, s\right)$ is defined as

$$
\left[\operatorname{det}\left(I_{14}-A_{v} \otimes B_{v} q_{v}^{-s}\right)\right]^{-1} .
$$

Theorem 2.1. Let $f(\tau, s$,$) and F$ be factorizable functions as defined above.

Let $S$ be the set of places $v$ where $v$ is Archimedean and where the local components $f_{v}\left(\tau_{v}, s,\right)$ and $F_{v}$ are not spherical vectors.

Let $\Pi$ be a "generic" representation. Then

$$
\mathrm{II}(F, f(\tau, s, \quad))=\mathrm{II}_{S}(F, f(\tau, s)) L^{s}\left(\Pi \otimes \tau, \frac{1}{2}+s\right),
$$

where $\mathrm{II}_{S}$ is a local Euler product (specified below) over the primes $v \in S$ and $L^{S}(\Pi \otimes \tau, 1 / 2+s)$ is the restricted $L$ function

$$
L^{S}(\Pi \otimes \tau, 1 / 2+s)=\prod_{v \notin S} L_{v}\left(\Pi_{v} \otimes \tau_{v}, 1 / 2+s\right) .
$$

Moreover the function $L^{S}(\Pi \otimes \tau, s)$ admits a meromorphic extension to $\mathbf{C}$.

Remark. Since the Eisenstein series $\mathscr{E}(f(\tau, s)$,$) is normalized$ by $L^{S}(\tau, s+3 / 2) L^{S}\left(\tau, \mathrm{Sym}^{2}, 2 s+1\right)$ then it should follow that the function $s \rightsquigarrow L^{S}(\Pi \otimes \tau, s)$ admits a finite number of poles!

By the well-known principle of uniqueness of Whittaker models the representation $\Pi_{v}$ can be embedded into $\operatorname{Ind}_{\left(U_{0}\right)_{v}}^{G_{v}}\left(\psi_{\left(U_{0}\right) v}\right)$. Similarly $\tau_{v}$ can be embedded into $\operatorname{Ind}_{N\left(k_{v}\right)}^{G_{2}\left(k_{v}\right)}\left(\psi_{v}\right)$ where $\psi_{v}$ is a nontrivial additive character on $\left.N=\left\{\left(\begin{array}{cc}1 & x \\ 0 & 1\end{array}\right) \mid x \in k_{v}\right\}\right)$. Using these embeddings we let $W_{v}\left(\tau_{v}, g, s\right)$ belong to $\operatorname{Ind}_{P_{v}}^{G_{v}}\left(\left.\tau_{v} \otimes 1\right|_{v} ^{s+2}\right)$ 
and $W_{F_{v}} \in \Pi_{v}$ be the corresponding Whittaker functions. Then we form the integral

$$
(*)_{v}(s)=\int_{V_{v} \backslash G_{v}} W_{F_{v}}(g) W_{v}\left(\tau_{v}, g, s\right) d g,
$$

where $V_{v}$ is the 4-dimensional subgroup of $\left(U_{0}\right)_{v}$ which is an extension of $Z_{v}$ by the 1-dimensional root subgroup corresponding to a long simple root of $G$.

In fact we have that the data is chosen in such a way that

$$
\mathrm{II}_{S}(F, f(\tau, s))=\prod_{v \in S}(*)_{v}(s) .
$$

Then we prove that

Corollary to Theorem 2.1. Let $s_{0} \in \mathrm{C}$ be given. Then it is possible to choose the data $F$ and $f(\tau, s$,$) (depending on s_{0}$ ) so that $\mathrm{II}\left(F(f(\tau, s))=,I_{\infty}(F, f(\tau, s),) L^{S}\left(\Pi \otimes \tau, \frac{1}{2}+s\right)\right.$ where $I_{\infty}$ is a meromorphic function in $s$ which is nonvanishing at $s_{0}$.

We note that we can extend the application of the method of Theorem 2.1 to cover another $L$ function for the group $G$. Rather than use a cusp form $\tau$ we use an Eisenstein series on the group $G L_{2}(\mathbf{A})$. Indeed we consider the parabolic subgroup $\widetilde{P}^{\prime}$ of $S O\left(\varphi_{\psi}\right)$ contained in $\widetilde{P}$ of the form $\widetilde{B}_{2} \cdot S O(3) \cdot \widetilde{U}$ where $\widetilde{B}_{2}$ is the Borel subgroup of $G L_{2}$. Then considering $T_{2} \subset B_{2}$ as a maximal torus, we define a character $\left(\delta(s, \mu)\right.$ on $T_{2}=\left\{\left(t_{1}, t_{2}\right) \in\right.$ $\left.k^{x} \times k^{x}\right\} \rightsquigarrow\left|t_{1}\right|^{s+\mu+5 / 2}\left|t_{2}\right|^{s-\mu+3 / 2}$. We form the family of induced automorphic representations

$$
I(\mu, s)=\operatorname{Ind}_{\widetilde{P}^{\prime}(\mathbf{A})}^{S O(Q)(\mathbf{A})}\left(\delta_{(s, \mu)} \otimes 1_{S O(3)} \otimes 1_{\widetilde{U}}\right) .
$$

With such a family we construct Eisenstein series. Let $f(s, \mu, x)$ be an analytic family in $I(\mu, s)$, and we form for appropriate $(s, \mu)$ the Eisenstein series

$$
\mathscr{E}(f(s, \mu, x))=\sum_{\widetilde{P}^{\prime}(k) \backslash S O(Q)(k)} f(s, \mu, \gamma x) .
$$

Again the normalizing factor for $\mathscr{E}(f(s, \mu, x))$ is

$$
\begin{aligned}
& L^{S}(1, s+\mu+3 / 2) L^{S}(1, s-\mu+3 / 2) L^{S}(1, s+\mu+1 / 2) \\
& \quad \times L^{S}(1, s-\mu+1 / 2) L^{S}(1,2 s+2 \mu) L^{S}(1,2 s-2 \mu) \\
& \quad \times L^{S}(1,2 s+1) L^{S}(1,2 \mu+1) \\
& \quad \equiv \xi^{S}(s, \mu) .
\end{aligned}
$$


Then we form again the Rankin-Selberg integral

$$
\mathrm{II}(F, f(s, \mu,))=\xi^{S}(s, \mu) \int_{G(k) \backslash G(\mathbf{A})} F(x) \mathscr{E}(f(s, \mu, x)) d x .
$$

Then we prove the analogue of Theorem 2.1.

Theorem 2.2. Let $F$ and $f(s, \mu$,$) be factorizable functions as$ given in Theorem 2.1. Let $S$ be also as given in Theorem 2.1 . Then

$$
\begin{aligned}
& \mathrm{II}(F, f(s, \mu,)) \equiv \mathrm{II}_{S}(F, f(s, \mu,)) \\
& \quad L^{S}(\Pi, s+\mu+1 / 2) L^{S}(\Pi, s-\mu+1 / 2)
\end{aligned}
$$

where $\mathrm{II}_{S}$ is a local Euler product over the primes in $S$ and $L^{S}(\Pi, \lambda)$ is the restricted $L$ function

$$
L^{S}(\Pi, \lambda)=\prod_{v \notin S} L_{v}\left(\Pi_{v}, \lambda\right) .
$$

Here $L_{v}$ is the local Euler factor defined in the following manner (see comments preceding Theorem 2.1)

$$
L_{v}\left(\Pi_{v}, \lambda\right)=\left[\operatorname{det}\left(I_{7}-A_{v} q_{v}^{-\lambda}\right)\right]^{-1} .
$$

Moreover the function $L^{S}(\Pi, \lambda)$ admits a meromorphic extension to $\mathbf{C}$.

Remark. We note that we can specialize the above Rankin-Selberg integral along a certain hyperplane $s-\mu+1 / 2=K_{0}$ (along which the Eisenstein series $\mathscr{E}(f(s, \mu)$,$) has no singularities) with K_{0}$ a real large integer. Then we have a Rankin-Selberg representation of $L^{S}(\Pi, \lambda)$. Note that $L^{S}(\Pi, s-\mu+1 / 2)=L^{S}\left(\Pi, K_{0}\right)$ is given by a convergent Euler expansion! We also expect as before that $L^{S}(\Pi, \lambda)$ has a finite number of poles in $\lambda$.

\section{UNRAMIFIED CASE AND BRANCHING FORMULA}

If the data is $(*)_{v}$ is unramified (i.e. the local components are spherical) then we calculate

\section{Lemma 3.1.}

$$
(*)_{v}(s)=\frac{L_{v}\left(\Pi_{v} \otimes \tau_{v}, \frac{1}{2}+s\right)}{L_{v}\left(\tau_{v}, S y m^{2}, 2 s H\right) L_{v}\left(\tau_{v}, s+\frac{3}{2}\right)} .
$$

The basis of such a calculation is the use of the CasselmanShalika formula for Whittaker functions in terms of characters of irreducible finite-dimensional representations. 
The critical step in this calculation is the use of a certain Branching Formula from $S O(7, \mathrm{C})$ with highest weight $\left(\lambda_{1}, \lambda_{2}, 0\right)$ (with $\lambda_{i} \in \mathbf{Z}$ and $\left.\lambda_{1} \geq \lambda_{2} \geq 0\right)$ and consider the restriction to $G_{2}(\mathbf{C})$.

We parametrize a highest weight representation of $G_{2}(\mathbf{C})$ by a pair $(m, n)$ (with $m, n \in \mathbf{Z}$ and $m \geq n \geq 0$ ), which corresponds to the weight $n \omega_{1}+(m-n) \omega_{2}$ with $\omega_{1}, \omega_{2}$ fundamental weights of $G_{2}(\mathbf{C})$ dual to the two simple roots $\alpha_{1}$ and $\alpha_{2}$. Then the particular Branching Formula we prove is the following:

$$
\left(\lambda_{1}, \lambda_{2}, 0\right)=\sum_{\alpha=0}^{\alpha=\min \left(\lambda_{1}-\lambda_{2}, \lambda_{2}\right)} \sum_{\beta=\alpha}^{\beta=\lambda_{2}}\left(\lambda_{1}-\alpha, \beta\right) .
$$

The formula is stated in [J]. Our proof is based on using the ideas of $[\mathrm{BGG}]$ and $[\mathrm{KV}]$.

\section{REFERENCES}

[BGG] J. Bernstein, I. Gelfand, and S. Gelfand, Models of representations of Lie groups, Selecta Math. Soviet 1 (2) (1981), 121-142.

[G-PS-R] S. Gelbart, I. Piatetski-Shapiro, and S. Rallis, Explicit constructions of automorphic L functions, Lecture Notes in Math., vol. 1254, SpringerVerlag, New York, 1989.

[GS] S. Gelbart and F. Shahidi, Analytic properties of automorphic L functions, Perspectives in Mathematics, Academic Press, Boston, Massachusetts, 1988.

[H] C. Herz, Alternating 3 forms and exceptional Lie groups of type $G_{2}$, Canad. Math. J. 35 (1983), 776-806.

[J-L] H. Jacquet and R. Langlands, Automorphic forms on $G L(2)$, Lecture Notes in Math., vol. 104, Springer-Verlag, New York, 1970.

[J] B. R. Judd, Atomic spectroscopic methods, Harcourt Brace, New York 1977.

[J-PS-Sh] H. Jacquet, I. Piatetski-Shapiro, and J. Shalika, Rankin-Selberg convolutions, Amer. J. Math., 105 (1983), 367-464.

[J-S-1,2] H. Jacquet and J. Shalika, On Euler products and classification of automorphic representations, I and II, Amer. J. Math. 103 (3) (1981), 499-558 and 777-815.

[KV] B. Kimelfeld and E. Vinberg, Homogeneous domains on flag manifolds and spherical subgroups of semisimple Lie groups, Functional Anal. Appl. 12 (3) (1978), 168-174.

[L] R. Langlands, Euler products, Whitmore Lectures, Yale Univ. Press, 1967.

[PS-R] I. Piatetski-Shapiro and S. Rallis, Rankin triple L-functions, Comp. Math. 64 (1987), 31-115.

[RS] S. Rallis and G. Schiffman, Theta correspondence associated to $G_{2}$, Amer. J. Math. (to appear). 
[W-1] A. Weil, Basic number theory, die Grundlehren der Mathematischen Wissenschaflen, vol. 144, Springer-Verlag, Berlin and New York, 1967.

[W-2] A. Weil, Uber die Bestimmung Dirichletscher Reihen durch funktionalgleichungen, Math. Ann. 168 (1967), 149-156.

YALE UNIVERSITY AND Tel-Aviv UNIVERSITY

The OHIo STATE UNIVERSity

Strasbourg UNIVERSITY 
